\title{
A Survey on Underwater Image Enhancement Techniques
}

\author{
Pooja Sahu \\ MTech Scholar Deptt.of ECE, \\ Truba Inst. of Engg. \& Info. Tech. \\ Bhopal
}

\author{
Neelesh Gupta \\ Deptt.of ECE, Truba Inst. of \\ Engg. \& Info. Tech. Bhopal
}

\author{
Neetu Sharma \\ Deptt.of ECE, Truba Inst. of \\ Engg. \& Info. Tech. Bhopal
}

\begin{abstract}
This review paper deals with the methods to improve underwater image enhancement techniques, the processing of underwater image captured is necessary because the quality of underwater images affect and these image leads some serious problems when compared to images from a clearer environment. A lot of noise occurs due to low contrast, poor visibility conditions (absorption of natural light), non uniform lighting and little color variations, pepper noise and blur effect in the underwater images because of all these reasons number of methods are existing to cure these underwater images different filtering techniques are also available in the literature for processing and enhancement of underwater images one of them is image enhancement using median filter which enhances the image and help to estimate the depth map and improve quality by removing noise particles with the help of different techniques, and the other is RGB Color Level Stretching have used. Forward USM technique can also be used for image enhancement.
\end{abstract}

\section{Keywords}

RGB Color Level, color enhancement, Light Correction Method, Median filter.

\section{INTRODUCTION}

Due to the poor visibility conditions the environment of the world's oceans is still not well explored for this purpose Underwater image enhancement techniques are used, because the earth is an aquatic planet and as the fact about $70 \%$ of its surface is covered by water. Now a days there is a strong interest in knowing what lies in underwater, and moreover, this field has made an importance to the use of underwater sequences to monitor marine species, Underwater mountains \& plants, to achieve this purpose it is absolutely necessary to use the clear images. Here also discussed the effects of color in underwater images. With respect to light reflection, Church [4] describes that the reflection of the light varies greatly depending on the structure of the sea. Another concern is related to the water that bends the light either to make crinkle patterns or to diffuse. Most importantly, the quality of the water controls and influences the filtering properties of the water such as sprinkle of the dust in water [12].

According to Anthoni [5] the reflected amount of light is partly polarized horizontally and partly enters the water vertically. An important quality of the vertical polarization is that it makes the object less shining and helps to capture deep colors which may not be possible to capture otherwise. Another well-known problem concerning the underwater images is related to the density of the water in the sea which is considered 800 times denser meadium than air. Therefore, when light rays moves from the air to the water, it is partly reflected reverse and at the same time partly enters the water [5]. The total light amount that enters the water also starts reducing start it goes deeper in the sea [6]. Similarly, the water molecules also absorb certain amount of light [12]. As a result, the underwater images are getting darker and darker as the deepness increases. Not only the quantity of light rays is condensed when it goes deeper but also colors drop off one by one depending on the wavelength of the colors. For example, first of all red color disappears at the depth of $3 \mathrm{~m}$. Secondly, orange color starts disappearing while we go further. At the depth of $5 \mathrm{~m}$, the orange color is lost. Thirdly most of the yellow goes off at the depth of $10 \mathrm{~m}$ and finally the green and purple disappear at further depth [12].

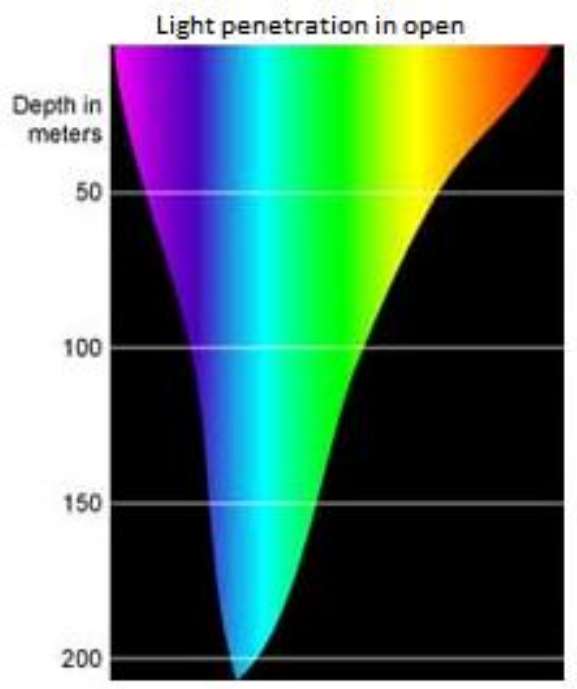

Fig 1- Color penetration pattern

From the fig1 it can be clearly understand that, the blue color travels the longest in the water and in depth due to its shortest visible wave length. Which makes the underwater images having been dominated only by blue color because of this effect of blue color the original color of any object under the water is affected. In addition to excessive amount of blue color, the blur images contain little brightness, little contrast and so on.

\section{LITERATURE SURVEY}

This section presents related literature concerning underwater image processing and enhancement techniques. In $1990 \mathrm{Wn}$. R Schneider proposed to use the colored filters in Black and White photography to change the way tones of a scene record on film. Yellow filters are commonly. Because the light coming from clouds is white, it is not affected by the filter like that from the blue sky. Hence a yellow filter causes clouds to stand out more than usual. It affects the quality of the image. This work was mainly for the images which are in the presence of air, and there were some need to be explore the underwater world too. 
In 2010 Iqbal, K.; Odetayo, M.; James, A.; Salam, R.A.; Talib, A.Z.H. [14] worked on "Enhancing the low quality images using Unsupervised Colour Correction Method,", The affected underwater images reduced contrast and non-uniform color cast because of the absorption and scattering of light rays in the marine environment. For that they proposed an Unsupervised Colour Correction Method (UCM) for underwater image quality enhancement. UCM is based on color matching, contrast improvement of RGB color model and contrast improvement of HSI color model. Firstly, the color cast is concentrated by equalizing the color values. Secondly, an improvement to a contrast alteration method is useful to increase the Red color by stretching red color histogram towards the utmost, similarly the Blue color is concentrated by stretching the blue histogram to the minimum. Thirdly, the Saturation and Intensity parts of the HSI color model have been useful for contrast correction to enlarge the true color using Saturation and to address the illumination problem through Intensity.

In 2011 Jinbo Chen; Zhenbang Gong; Hengyu Li; Shaorong Xie, [15] proposed "A detection method based on sonar image for underwater pipeline tracker,". The surveillance and inspection of underwater pipelines are carried out by operators who drive a remotely operated underwater vehicle (ROV) with camera mounted on it. Though in extremely turbid water, the camera cannot capture any scene, even with supplementary high-intensity light. In this case the optical detection devices are unable to complete the surveillance task In recent years, forward looking sonar is broadly applied to the underwater examination, which is not subject to the control of light and turbidity. So it is appropriate for the inspection of pipelines. But the active change of ROV by the water flow will show the way to the aim to escape from the sonar image effortlessly. In adding up, the sonar image is with high noise and little contrast. It is difficult for the operator to identify the pipeline from the images. Furthermore, the observation of underwater pipelines is deadly and time unbearable and it is easy to create mistakes due to the exhaustion and interruption of the operator. Then, the study focuses on rising image processing algorithms to distinguish the pipeline repeatedly. By means of the proposed image processing technique, firstly the images are improved using the Gabor filter. And then these images are useful for an edge detector. Lastly the parameters of the pipeline are designed by Hough transform. To decrease the search area, the Kalman filter is explored to forecast the parameters of the pipeline on the next picture. And the research is shown the vision system is on hand to the observation of underwater pipelines.

In 2011 Hung-Yu Yang; Pei-Yin Chen; Chien-Chuan Huang; Ya-Zhu Zhuang; Yeu-Horng Shiau,[16] worked on "Low Complexity Underwater Image Enhancement Based on Dark Channel Prior,". Blurred underwater image is always an irritating problem in the deep-sea engineering. They proposed an competent and low complexity underwater image enhancement technique based on dark channel before. Our technique employs the median filter in its place of the soft matting method to estimate the depth map of image. Furthermore, a color improvement method is adopted to improve the color contrast for underwater image. The tentative results show that the proposed approach can well improve the underwater image and decrease the implementation time. In addition, this technique requires fewer computing reserve and is well appropriate for implementing on the supervision and underwater navigation in real time.

In 2012 Chiang, J.Y.; Ying-Ching Chen, [17] researched on "Underwater Image Enhancement by Wavelength Compensation and Dehazing,". Where light scattering and color modify are two main sources of alteration for underwater shooting. Light scattering is affected by light event on objects reflected and deflected many times by particles present in the water prior to reaching the camera. This in turn lowers the visibility and contrast of the image captured. Color change corresponds to the unstable degrees of reduction encountered by light traveling in the water with diverse wavelengths, depiction ambient underwater environments conquered by a bluish quality. No obtainable underwater processing techniques can handle light dispersion and color change distortions caused by underwater images, and the probable presence of false lighting concurrently. This literature proposed a novel systematic come up to to improve underwater images by a de-hazing algorithm, to give back the attenuation difference along the broadcast path, and to take the pressure of the possible presence of an false light source into consideration. Previously the deepness map, i.e., distances between the objects and the camera, is expected, the foreground and background within a view are segmented. By managing the effect of artificial light, the haze occurrence and inconsistency in wavelength attenuation along the underwater broadcast path to camera are corrected. Secondly, the water deepness in the image scene is predictable according to the remaining energy ratios of diverse color channels obtainable in the background light. In 2012 bt. Shamsuddin, N.; bt. Wan Ahmad, W.F.; Baharudin, B.B.; Kushairi, M.; Rajuddin, M.; bt. Mohd, F.,[18] developed a technique on "Significance level of image enhancement techniques for underwater images,". Underwater imaging is fairly a demanding in the area of photography specially for low resolution and normal digital camera. There are some problems arise in underwater images such as partial range visibility, low contrast, non identical lighting, blurring, intense artifacts, color diminish and noise. This research concentrated on color diminished. Major application of typical computer vision techniques to marine imaging is mandatory in dealing with the thought problems. Both automatic and manual level methods are used to record the mean values of the stretched histogram.

In 2013 Hitam, M.S.; Yussof, W.N.J.H.W.; Awalludin, E.A.; Bachok, Z., [19] has been worked on "Mixture contrast limited adaptive histogram equalization for underwater image enhancement,". By improving the quality of an underwater image has received substantial attention due to rundown visibility of the image which is caused by physical properties of the water. Here they presented a new technique called hybrid Contrast Limited Adaptive Histogram Equalization (CLAHE) color spaces that specifically developed for underwater image improvement. The technique operates CLAHE on RGB and HSV color spaces and both results are joint together using Euclidean rule. Tentative results show that the future approach considerably improves the visual quality of underwater images by enhancing contrast, as well as dropping noise and artifacts. 
Table 1: Summary of Literature Review

\begin{tabular}{|c|c|c|c|c|}
\hline Year & Author & Title & Apprach & Result \\
\hline 2010 & $\begin{array}{l}\text { Iqbal, K.; Odetayo, M.; James, } \\
\text { A.; Salam, R.A.; Talib, A.Z.H. }\end{array}$ & $\begin{array}{c}\text { Enhancing the low quality images } \\
\text { using Unsupervised Color Correction } \\
\text { Method }\end{array}$ & $\begin{array}{c}\text { Unsupervised Color } \\
\text { Correction Method (UCM) }\end{array}$ & $\begin{array}{c}\text { Enhanced Illumination } \\
\text { and Contrast }\end{array}$ \\
\hline 2011 & $\begin{array}{l}\text { Jinbo Chen; Zhenbang Gong; } \\
\text { Hengyu Li; Shaorong Xie }\end{array}$ & $\begin{array}{l}\text { A detection method based on sonar } \\
\text { image for underwater pipeline tracker }\end{array}$ & Sonar image detection method & $\begin{array}{c}\text { Good in extreme turbid } \\
\text { water }\end{array}$ \\
\hline 2011 & $\begin{array}{l}\text { Hung-Yu Yang; Pei-Yin Chen; } \\
\text { Chien-Chuan Huang; Ya-Zhu } \\
\text { Zhuang; Yeu-Horng Shiau }\end{array}$ & $\begin{array}{c}\text { Low Complexity Underwater Image } \\
\text { Enhancement Based on Dark Channel } \\
\text { Prior }\end{array}$ & Dark Channel Method & $\begin{array}{c}\text { Decrease in } \\
\text { implementation time }\end{array}$ \\
\hline 2012 & Chiang, J.Y.; Ying-Ching Chen & $\begin{array}{c}\text { Underwater Image Enhancement by } \\
\text { Wavelength Compensation and } \\
\text { Dehazing }\end{array}$ & $\begin{array}{l}\text { Wavelength Compensation } \\
\text { and Dehazing }\end{array}$ & $\begin{array}{l}\text { De-hazing and } \\
\text { improvement in quality } \\
\text { in deep water }\end{array}$ \\
\hline 2012 & $\begin{array}{l}\text { bt. Shamsuddin, N.; bt. Wan } \\
\text { Ahmad, W.F.; Baharudin, B.B.; } \\
\text { Kushairi, M.; Rajuddin, M.; bt. } \\
\text { Mohd, F. }\end{array}$ & $\begin{array}{l}\text { Significance level of image } \\
\text { enhancement techniques for } \\
\text { underwater images }\end{array}$ & $\begin{array}{l}\text { Based on Color Diminished } \\
\text { and Stretched }\end{array}$ & $\begin{array}{l}\text { Having better marine } \\
\text { imaging applications }\end{array}$ \\
\hline 2013 & $\begin{array}{l}\text { Hitam, M.S.; Yussof, } \\
\text { W.N.J.H.W.; Awalludin, E.A.; } \\
\text { Bachok, Z. }\end{array}$ & $\begin{array}{c}\text { Mixture contrast limited adaptive } \\
\text { histogram equalization for underwater } \\
\text { image enhancement }\end{array}$ & $\begin{array}{c}\text { Adaptive Histogram } \\
\text { Equalization by Enhancing } \\
\text { contrast, as well as dropping } \\
\text { noise and artifacts }\end{array}$ & $\begin{array}{l}\text { Improves the visual } \\
\text { quality of underwater } \\
\text { images }\end{array}$ \\
\hline
\end{tabular}

\section{UNDERWATER IMAGE ENHANCEMENT}

Image enhancement is a technique of improving the quality of image by improving its feature and its RGB values.

The underwater image processing area has received considerable attention within the last decades, showing important achievements. This paper has a review on some of the most recent methods that have been specifically developed for the underwater scenarios. These methods are capable of extending the range of underwater image processing, improving image contrast level and resolution quality. After consideration of the basic physics of the light propagation in the water medium, we focus on the different methods available in the previous articles. The situation for which all of them have been firstly developed are highlighted as well as the quality assessment methods used to evaluate their perform ance.

A major difficulty to process underwater images comes from light attenuation, it limits the visibility distance, at about twenty meters in clear water and five meters or less in muddy water. The light reduction process is caused by the absorption (which removes light energy) and spreading (which changes the direction of light wave path). Absorption of light and its scattering effects are because of the water itself and to other components such as dissolved organic matter or small observable floating particles. Due to this difficulty, underwater imaging suffers too many problems [1][4]:

first the quick attenuation of light requires attaching a light source to the vehicle providing the essential lighting. Unluckily, synthetic lights tend to illuminate the scene in a non uniform fashion producing a bright spot in the center of the image and poorly illuminated area surrounding. Then the distance between the camera and the scene usually induced prominent blue or green color (the wavelength corresponding to the red color disappears in only few meters). Then, the floating particles highly variable in kind and application, boost absorption and dispersion effects: they blur image features (advance scattering), transform colors and turn out glow artifacts known as "marine snow". Lastly the non solidity of the sunken vehicle affects once again image contrast. From the pre-processing filter has been assessed on natural underwater images with and without additional synthetic underwater degradations as proposed in [1]. Underwater perturbations we added are typical perturbations observed and it have been tested with varying degrees of severity. We simulate blur and unequal illumination using Jaffe and McGlamery's model [9][11], gaussian and particles noise as additive contributions to the images and finally reduced color range by histogram operation.

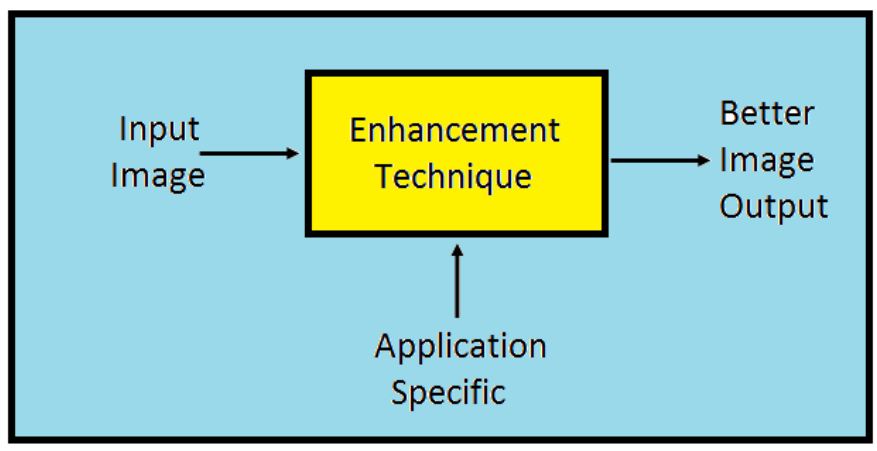

Fig 2: Basic block diagram of image enhancement

Fig2 shows the basic diagram of image enhancement process in which the specific enhancement technique can be any one of which discussed above. Gaussian Blur and Log-Gabor. In the first occurrence, they applied histogram equalization to eradicate back scattering, attenuation and lighting effect. Applying the contrast histogram methods does not promise the elimination of noise in the images. to address this issue, they additionally use Gaussian blur method, a low pass filtering method. In fact, they pick two images from unique image using partition and subtraction. After mixture, the left over noise is removed using multi-scale de-noising algorithm based on complex valued Log Gabor wavelets [2]. Similarly Fair weather [3] have used 
techniques such as contrast stretching and Markov Random field. They apply the bimodal histogram form to the images in order to increase the underwater image, firstly they use contrast stretching method. Next, they separated the image into two parts; object and background and then applied Markov Random field segmentation method. Yoav [8] have used a Physicsbased form.

\section{NOISE FILTERING}

Filtering is a process of removing the noisy elements which are present in digital images which are horizontal to a variety of noise. Noise is the result of errors in the image acquisition process that result in pixel values that do not reflect the true intensities of the real scene. There are several ways that remove the noise which introduced in an image, depending on how the image is created. Such as blur effect, pepper noise etc.

There are various ways in terms of filters to remove these noise two of them are:

(a) Removing noise through Linear filter:

The linear filtering can be used to remove certain noise types. Some filters, for instance averaging or Gaussian filters, are suitable for this reason, e.g., an averaging filter is helpful for removing grain noise from a snap. As each pixel gets situate to the common of the pixels in its surrounding area, local changes caused by particle are compact.

(b) Removing noise through median filter:

Underwater image enhancement is also done with the help of one median filter [13] an efficient and low complexity underwater image enhancement method which contains two method one is the Median filter which is used to approximation the transmission of input image. The atmospheric light $\mathrm{A}$ is obtained by using dark channel prior. Further upgrading a color correction quality is employed to enhance the color contrast of the object in underwater. Median filtering is similar to using an averaging filter, in that every output pixel is put to an average of the pixel values in the neighborhood of the corresponding input pixel. Though, by median filtering, the value of an output pixel is determined by the median of the region pixels, somewhat than the mean. The median is greatly less responsive than the mean to great values. Median filtering is consequently well able to eliminate these outliers without declining the unevenness of the image.

(c) Photographic Unsharp Masking:

The method was initially used in Germany during the 1930 s as a way of increasing the acutance, or apparent resolution, of photographic images.

For the photographic process, a large-format glass plate negative is contact-copied onto a low contrast film or plate to create a positive image. Though, the positive copy is prepared with the copy stuff in contact with the reverse of the unique, rather than mixture-to mixture, so it is distorted. After processing this blurred positive is replaced in contact with the back of the original negative. After light is passed throughout equally negative and inrecord positive, the positive somewhat cancels a few of the information in the negative.

Because the positive has been blurred intentionally, only the low frequency (blurred) information is cancelled. As well, the mask efficiently reduces the vibrant range of the original negative. Therefore, if the ensuing increased size image is recorded on contrasts pictorial paper, the fractional deletion emphasizes the high occurrence information in the original, exclusive of loss of emphasize or shadow aspect. The consequential print appears more sensitive than one made lacking the unsharp mask: its acutance is enlarged. In the pictorial procedure, the quantity of blurring can be restricted by altering the "softness" or "hardness of the light source used for the first unsharp mask revelation, while the strength of the effect can be controlled by changing the contrast and density (i.e., exposure and development) of the unsharp mask. For traditional photography, unsharp masking is usually used on monochrome materials; special panchromatic soft-working black and white films have been available for masking photographic color transparencies. It is particularly useful to manage the density range of a transparency intended for photomechanical reproduction.

USM can increase either sharpness or (local) contrast because these are both forms of increasing differences between values, increasing slope - sharpness referring to very small-scale (high frequency) differences, and contrast referring to larger scale (low frequency) differences. Extra dominant methods for improving tone are referred as tone mapping.

\section{PROBLEM STATEMENT}

This review paper covers the image enhancement techniques and the image quality enhancement using filers, the atmospheric light is a major difficulty to process underwater images comes from the poor visibility conditions under the water, scattering of light and light attenuation due to all the reasons the underwater images suffers a lot and affect their visibility and the contrast which they contain actually. Light attenuation limits the visible distance, at about 20 meters in clear water and 5 meters or less in turbid or less muddy water.

Use of median filter which has proposed by Hung- Yu Yang, Pie-Yin Chen[13] which is used to estimate the transmission of input image the atmospheric light is obtained by using dark channel prior and used to remove the noise like pepper noise, with this method the noise can be removed and the image which has less amount of noise and more improved image can be achieved but the actual color contrast and less sharp image is less accurate than the original image therefore in future there is a need of some method in addition to improve the quality of these kind of underwater images.

\section{CONCLUSIONS}

In this review paper, we focused on a survey of different techniques of underwater image enhancement to enhance the quality of underwater images and different techniques used Color Stretching \& USM filter both on RGB to enhance underwater images. The approached used i.e. median filter [13] which is used to estimate the transmission of input image. The atmospheric light is obtained by using dark channel prior. Further improvement a color correction quality is employed to enhance the color contrast of the object in underwater and remove different noise particles. 
In order to increase the efficiency of underwater images and improve the quality and to get more sharper and accurate images, as per the study in the future we can work in the sequence of the existing methods for underwater images make more clearer and sharper that the use USM filtering technique. An USM not creates extra facts, but it can really improve the look of details by increasing small scale acutance and get the output image more improved by applying two stages of USM which will help to reduce complexity of the system and also addresses the problem of color levels. In future this work will help in other applications where smoky, blurry etc image environments.

\section{REFERENCES}

[1] M. Chambah, A. Renouf, D. Semani, P. Courtellemont A. Rizzi, "Underwater colour constancy: enhancement of automatic live fish recognition" 2004, In Electronic Imaging.

[2] Andreas Arnold-Bos, Jean-Philippe Malkasse and illes Kervern: "Towards a model-free denoising of underwater optical images" In IEEE Conference on Oceans, 2005.

[3] A J R Fair weather, M A Hodgetts, A R Greig, "Robust scene interpretation of underwater image sequences", In 6th International Conference on Image Processing and its Applications, 1997, pp. 660 -664, ISBN: 085296692 X.

[4] White, E.M., Partridge, U.C., Church, S.C, "Ultraviolet dermal reflection and mate choice in the guppy", In 2003, pp. 693-700.

[5] J Floor Anthoni 2005.

[6] Balvant Singh , Ravi Shankar Mishra , Puran Gour (IJCTEE)vol-1Issue-2, 2012.

[7] Cufi, X., Garcia, R., and Ridao, P. "An Approach To Vision-Based Station Keeping For An Unmanned Underwater Vehicle". Available via: IEEE/RSJ International Conference on Intelligent Robots and Systems (IROS), 2002.

[8] Schechner, Y and Karpel, N., "Clear Underwater Vision". Proceedings of the IEEE CVPR, Vol. 1, 2004, pp. 536-543.

[9] Garcia, R., Nicosevici, T., and Cufí, X., "On The Way to Solve Lighting Problems in Underwater Imaging", Proceedings of the IEEEOCEANS Conference (OCEANS), 2002, pp. 1018-1024.

[10] A. Rizzi, C. Gatta and D. Marini, "A New Algorithm for Unsupervised Global and Local Color Correction" pattern Recognition Letter, Vol. 24, no. 11, pp. 1663-1677,2003.
[11] R. Fisher, S. Perkins, A. Walker, E. Wolfart (2003), "Contrast Stretching".

[12] Luz Abril Torres-Méndez and Gregory Dudek, "Color Correction of Underwater Images for Aquatic Robot Inspection" Lecture Notes in Computer Science 3757, Springer A. Rangarajan, B.C. Vemuri, A.L. Yuille (Eds.), 2005, pp. 60-73, ISBN:3-540-30287-5.

[13] Hung-Yu, Pie-Yin Chen, Chien-Chuan Huang and Ya-Zhu Zhuang second International Conference on Innovations in Bio computing and Applications.

[14] Iqbal, K.; Odetayo, M.; James, A.; Salam, R.A.; Talib, A.Z.H., "Enhancing the low quality images using Unsupervised Colour Correction Method," Systems Man and Cybernetics (SMC), 2010 IEEE International Conference on , vol., no., pp.1703,1709, 10-13 Oct. 2010.

[15] Jinbo Chen; Zhenbang Gong; Hengyu Li; Shaorong Xie, "A detection method based on sonar image for underwater pipeline tracker," Mechanic Automation and Control Engineering (MACE), 2011 Second International Conference on , vol., no., pp.3766,3769, 15-17 July 2011.

[16] Hung-Yu Yang; Pei-Yin Chen; Chien-Chuan Huang; YaZhu Zhuang; Yeu-Horng Shiau, "Low Complexity Underwater Image Enhancement Based on Dark Channel Prior," Innovations in Bio-inspired Computing and Applications (IBICA), 2011 Second International Conference on , vol., no., pp.17,20, 16-18 Dec. 2011.

[17] Chiang, J.Y.; Ying-Ching Chen, "Underwater Image Enhancement by Wavelength Compensation and Dehazing," Image Processing, IEEE Transactions on, vol.21, no.4, pp.1756,1769, April 2012.

[18] bt. Shamsuddin, N.; bt. Wan Ahmad, W.F.; Baharudin, B.B.; Kushairi, M.; Rajuddin, M.; bt. Mohd, F., "Significance level of image enhancement techniques for underwater images," Computer \& Information Science (ICCIS), 2012 International Conference on, vol.1, no., pp.490,494, 12-14 June 2012.

[19] Hitam, M.S.; Yussof, W.N.J.H.W.; Awalludin, E.A.; Bachok, Z., "Mixture contrast limited adaptive histogram equalization for underwater image enhancement," Computer Applications Technology (ICCAT), 2013 International Conference on, vol., no., pp.1,5, 20-22 Jan. 2013.

\section{AUTHOR'S PROFILE}

Pooja Sahu is research scholar at Truba Institute of Engineering in Department of Electronics and Communication Engineering pursuing M.Tech. in Digital Communication. Areas of research are Digital Image Processing and Image Enhancement 